\title{
Interim results from ongoing Phase III placebo-controlled, randomized trial of hepcortespenlisimut-L for advanced hepatocellular carcinoma indication
} \author{
Reid $^{6}$, Vika Borisova ${ }^{6}$, Allen I. Bain ${ }^{1}$, Vichai Jirathitikal ${ }^{1}$

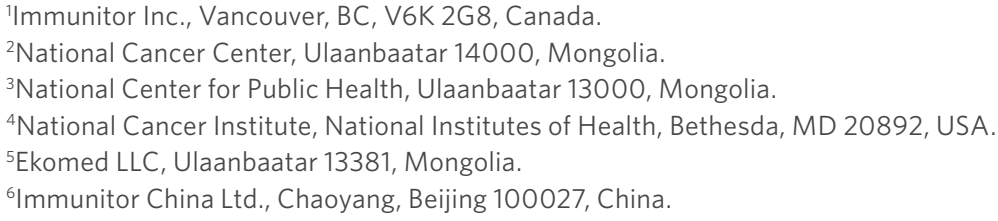

Aldar S. Bourinbaiar¹, Jigjidsuren Chinburen², Purev Batchuluun², Chogsom Munkhzaya², Dandii Oyungerel $^{3}$, Dorjiin Dandiï, Galyna A. Kutsyna ${ }^{4}$, Hulan Tsogkhuu ${ }^{5}$, Marina G. Tarakanovskaya ${ }^{5}$, Alan A.

Correspondence to: Dr. Aldar Bourinbaiar, Immunitor Inc., 365-2912 West Broadway, BC, V6K 2G6, Canada. E-mail: aldar@immunitor.com

\begin{abstract}
How to cite this article: Bourinbaiar AS, Chinburen J, Batchuluun P, Munkhzaya C, Oyungerel D, Dandii D, Kutsyna GA, Tsogkhuu H, Tarakanovskaya MG, Reid AA, Borisova V, Bain Al, Jirathitikal V. Interim results from ongoing Phase III placebo-controlled, randomized trial of hepcortespenlisimut-L for advanced hepatocellular carcinoma indication. Hepatoma Res 2020;6:2. http://dx.doi.org/10.20517/2394-5079.2019.25
\end{abstract}

Received: 30 Oct 2019 First Decision: 18 Nov 2019 Revised: 23 Dec 2019 Accepted: 23 Dec 2019 Published: 7 Jan 2020

Science Editor: Guang-Wen Cao Copy Editor: Jing-Wen Zhang Production Editor: Tian Zhang

\begin{abstract}
Aim: We aimed to further investigate the role of hepcortespenlisimut-L (Hepko-V5 or V5), a new oral immunotherapy developed by us, for hepatocellular carcinoma (HCC) indication.

Methods: The interim data from ongoing Phase III placebo-controlled, randomized trial were evaluated on the initial group of patients in advanced stage of HCC with emphasis on liver function and tumor marker alpha-fetoprotein levels. Additionally, an in vitro study was undertaken to elucidate the mechanism of action of $\mathrm{V} 5$ by measuring with flow cytometry the expression of cytokines such as IL-2, INF- $\gamma$, and TNF- $\alpha$ and cell activation markers CD69 and Ki67 on CD4- and CD8-positive lymphocytes isolated from peripheral blood of healthy volunteers.
\end{abstract}

Results: As early as one month after treatment initiation, there was a clear improvement in alanine transaminase, aspartate transaminase, alkaline phosphatase, and bilirubin levels among HCC patients who received daily dose of V5, but not in the placebo group. Additionally, alpha-fetoprotein (AFP) levels among V5 recipients decreased, while in the

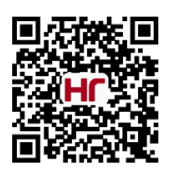


placebo group they rose. Clinical results are in line with in vitro observations indicating immune activation, as evidenced by many-fold enhancement of CD69, Ki67, and INF- $\gamma$ expression and at the same time marked anti-inflammatory effect resulting in 10-fold decrease in TNF- $\alpha$ output and lack of influence on IL-2 production.

Conclusion: Hepcortespenlisimut- $L$, a tableted oral formulation derived from heat-inactivated pooled blood of patients with HCC and viral hepatitis shows beneficial clinical effect, as demonstrated by improvement in liver function and reduction of tumor marker AFP levels. These correlate with in vitro observations showing potent activation of the immune response and pronounced oral tolerance effect.

Keywords: Hepatocellular carcinoma, alpha-fetoprotein, alanine transaminase, aspartate transaminase, IL-2, INF- $\gamma$, TNF- $\alpha$, CD69, Ki67, CD4, CD8, T lymphocytes

\section{INTRODUCTION}

Primary liver tumors originate from hepatocytes, cholangiocytes, and mesenchymal cells. Hepatoma is generally defined as a cancer originating from liver cells. It is often called hepatocarcinoma or hepatocellular carcinoma (HCC). It is the most common cancer of the liver, ranking as the second leading cause of death from all types of cancers worldwide ${ }^{[1]}$. Multiple risk factors have been identified including chronic viral hepatitis, liver cirrhosis, non-alcoholic fatty liver disease (NASH), lifestyle factors comprising alcohol abuse and smoking, metabolic diseases such as diabetes and obesity, environmental toxins such as aflatoxin, and occasionally genetic and hereditary disorders ${ }^{[2]}$. While the development of HCC is complex, there is a consensus that the underlying cause is chronic inflammatory damage ${ }^{[3]}$. Conventional treatment with chemotherapy including sorafenib, regorafenib, lenvatinib, and cabozantinib is usually directed at killing cancer cells - an approach that is quite toxic, leading to liver damage ${ }^{[4]}$. This in turn leads to hepatoma recurrence. Ideally, an effective HCC treatment must be two-pronged: in addition to destroying malignant liver cells, normal liver cells must be spared from hepatotoxicity caused by treatment. Immunotherapies are generally regarded as less toxic, although these toxicities can be life-threatening if not managed promptly ${ }^{[5]}$. The FDA has recently approved three antibody-based drugs: nivolumab, pembrolizumab, and ramucirumab. Additional immune interventions from the checkpoint inhibitor PD family, i.e., durvalumab, atezolizumab, and bevacizumab, as well as tremelimumab, which belongs to the anti-CTLA4 class, are being investigated ${ }^{[6]}$. Experience has shown that none of the currently approved HCC drugs are free from adverse side effects, albeit with varying degrees of severity.

Hepcortespenlisimut-L (also known as Hepko-V5 or V5) is the immunotherapeutic drug from a novel class that has been through Phase II trial ${ }^{[7]}$. Anti-HCC property of V5 was discovered accidentally about ten years ago during clinical use for original primary indications, which are chronic viral hepatitis and liver cirrhosis $^{[8,9]}$. V5 has been commercially available since 2002, has been used by over 30,000 individuals, and has never been reported of causing any serious adverse reactions. The US FDA granted Hepko-V5 orphan drug designation status in 2014. The present paper provides preliminary data from the currently ongoing Phase III trial and summarizes some of unpublished studies.

\section{METHODS}

\section{Patients and treatment regimen}

This Phase III placebo-controlled, 1:1 randomized trial was initiated with the goal of recruiting a total of 120 patients with advanced HCC. The preliminary data from the initial batch of 30 enrolled patients are provided to evaluate whether observed results confirm the data from an earlier published Phase II open-label trial ${ }^{[7]}$. All patients were in advanced stage of HCC and unfit for standard therapy, i.e., surgical intervention, due to tumor size, its location, or multiplicity. Many patients had single or multiple events of recurrent lesions after surgical intervention, such as resection, transarterial embolization, radiofrequency 
ablation, percutaneous ethanol injection, or their combinations. At the presentation, all patients were either under palliative care or pronounced incurable without available treatment options - underlining the disease gravity. As the overwhelming majority of our HCC patients were in terminal stage of the disease, they often presented with a variety of symptoms related to decompensated cirrhosis including ascites, edema, variceal bleeding, portal thrombi, and hepatic encephalopathy. Besides these symptoms, abdominal discomfort and less frequently pain were common. Other encountered complaints were fatigue or weakness, cachexia, anorexia, skin itch, bleeding from gums and nose, vomiting, and jaundice. As no other intervention options except palliative care were available, patients consented to receive a once-per-day pill of Hepko-V5 $(n=14)$ or identically appearing placebo pill $(n=16)$ with follow-up lab tests at Months 1 and 2. Patients were instructed to take one pill before bedtime and come back after running out of pills after 30 days. Patients had baseline measurement for alpha-fetoprotein and standard liver function tests such as alanine transaminase (ALT), aspartate transaminase (AST), alkaline phosphatase, and total bilirubin. Additional tests included creatinine and prothrombin time. In such a way, we had a cohort representative of the real-life situation in Mongolia, where almost every patient is diagnosed only after symptoms of the disease become apparent or when tumors recur.

\section{V5}

V5 is derived from pooled blood of patients with HCC who are often viral hepatitis C, hepatitis B, and Delta virus carriers by employing proprietary technology developed by us. The process of manufacturing has been described in detail previously: it involves heat and chemical inactivation with subsequent formulation into a tablet capable of withstanding digestive degradation in the stomach $^{[9]}$. The principle for mechanism of action of V5 is not much different from established principles with old-fashioned killed vaccines, e.g., hepatitis B vaccine made from pooled plasma. V5 has been approved in Mongolia as a biologically active product since 2008. V5 Immunitor is presented as an $850 \mathrm{mg}$ coated pink pill, with 30 pills per one package. The recommended dose is one to two pills per day, and the dose being used in this study is one pill per day. The preparation is stable at ambient room temperature for at least three years.

\section{Flow cytometry analysis}

Peripheral blood mononuclear cells (PBMCs) from the blood of healthy donors were obtained by density gradient centrifugation. Lymphocytes were isolated from PBMCs using anti-human CD4 or CD8 magnetic MACS microbeads following the manufacturer's protocol (Miltenyi Biotec, Somerville, MA 02143, USA). Obtained cells were incubated with a crushed pill of V5 diluted one million-fold $\left(10^{-6}\right)$ in saline for $48 \mathrm{~h}$ and then subjected to fluorescence-activated cell sorter (FACS) analysis using a FACSCalibur flow cytometer (BD Biosciences, San Jose, CA). V5-exposed and control cells were stained with antibodies against CD3, CD4, CD8, IFN- $\gamma$, TNF- $\alpha$, and IL-2 obtained from BD Biosciences. Briefly, cells $\left(1 \times 10^{6}\right)$ were stained either with anti-CD4 or -CD8 and anti-CD69 or -Ki67 (BD Pharmingen). Labeled cells were fixed with 0.5\% paraformaldehyde and analyzed using FlowJo software (Tree Star Inc., Ashland, OR).

\section{Statistical analysis}

The primary endpoint for this study was the effect of Hepko-V5 on serum AFP levels as a surrogate marker of tumor burden changes. Secondary objectives were safety, adverse effects, and changes in liver function parameters, creatine levels, and pro-thrombin time. The difference between pre- and post-treatment parametric values was assessed by paired Student's $t$-test and linear regression analysis. Wilcoxon matched pairs ranking test and contingency table analysis were employed for categorical data using the statistical calculator GraphPad, which is freely available online (GraphPad Software, 2365 Northside Dr., San Diego, CA 92108, USA). The significance level for all tests was set at $P \leq 0.05$.

\section{Ethics approval and written consent}

All patients were explained benefits and risks of the intervention and provided informed written consent agreeing to participate. The study was approved by the Institutional Review Board of Immunitor LLC 
Table 1. Interim analysis of select data from ongoing Phase III trial in 30 advanced-stage HCC patients in Mongolia

\begin{tabular}{|c|c|c|c|c|c|c|}
\hline \multirow{2}{*}{ Parameters } & \multicolumn{3}{|c|}{ Hepcortespenlisimut-L $(n=14)$} & \multicolumn{3}{|c|}{ Placebo $(n=16)$} \\
\hline & Entry level & Month $1(P)$ & Month $2(P)$ & Entry level & Month $1(P)$ & Month $2(P)$ \\
\hline$\overline{A L T}(I U / L)$ & 68.1 & $36.2(0.01)$ & $34.4(0.002)$ & 51.2 & $44.8(0.51)$ & $50.3(0.93)$ \\
\hline AST (IU/L) & 89.3 & $52.7(0.002)$ & $48.9(0.006)$ & 72.5 & $77.9(0.71)$ & $76.6(0.79)$ \\
\hline $\operatorname{ALP}(I U / L)$ & 151.2 & $111.3(0.15)$ & $89.4(0.06)$ & 160.1 & $184.7(0.49)$ & $221.7(0.23)$ \\
\hline Bilirubin $(\mu \mathrm{M} / \mathrm{L})$ & 30.9 & $15.4(0.002)$ & $13.1(0.0008)$ & 28.1 & $17.8(0.1)$ & $25.3(0.8)$ \\
\hline $\operatorname{AFP}(I U / m L)$ & $9,619.4$ & $7,649.3(0.65)$ & $6,994.9(0.69)$ & $8,285.4$ & $11,340.9(0.55)$ & $23,157.8(0.6)$ \\
\hline
\end{tabular}

The values are expressed as means with $P$ values shown in parentheses, where appropriate. $P$ values shown for these parameters were derived from Student- $t$ test calculation. $P$ values for this parameter were derived from Wilcoxon ranking test; the difference between outcomes in AFP levels at study conclusion between two treatment arms at Month 2, i.e., 6994.9 vs. 23,157.8, is highly significant ( $P<$ 0.0001; Chi-square). HCC: hepatocellular carcinoma; AFP: alpha-fetoprotein; ALT, alanine transaminase; AST, aspartate transaminase; ALP, alkaline phosphatase

(\#IRB00010671) and conducted in accordance with the declaration of Helsinki in conformity with good clinical practice as defined by the International Conference on Harmonization. This study is registered at ClinicalTrials.gov site with ID: NCT02232490.

\section{RESULTS}

The interim analysis of data from Phase III placebo-controlled study is summarized in Table 1. At the time of interim analysis, only 30 patients randomized into two arms, with 14 patients in V5 arm and 16 patients in the placebo arm, were available for statistical analysis. The total planned number of patients to be enrolled into this trial is 120 . As the study is ongoing, the codes were not broken open; thus, the statistician had no access to information as to which arm received experimental drug or placebo. Nevertheless, there is a clear difference between arms starting as early as one-month post-treatment. Pills that were stamped with "1" (experimental drug; V5) had highly significant effect on measured liver function parameters, i.e., ALT, AST, total bilirubin, and alkaline phosphatase. In contrast, pills stamped with " 5 " (placebo) had no significant effect. Pills " 1 " had a very distinct effect on liver function improvement and this trend is consistent with AFP changes, while 5-labeled pills were not able to arrest AFP accrual, which increased almost three-fold, i.e., from 8285 to $23,158 \mathrm{IU} / \mathrm{mL}$. The patients who received hepcortespenlisimut-L experienced a decline in AFP levels, from 9619.4 to $6994.9 \mathrm{IU} / \mathrm{mL}$ at the end of the second month and the difference between the outcomes of the two arms was statistically significant $(P<0.0001$ by ChiSquare). Nevertheless, $P$ values for AFP within both arms did not reach significance levels due to wide inter-individual fluctuation of AFP levels at baseline ranging three or more orders of magnitude. Thus, the Wilcoxon test had insufficient power. However, when the evolution in AFP trend was compared in V5 vs. placebo arms, this discrepancy became highly statistically significant, which makes us believe that the outcome of the trial will be successful, in line with numerous other V5 trials for unrelated clinical indications. Data were analyzed on intent-to-treat fashion without excluding missing values, deaths, and non-compliance. These preliminary findings indicate that the batch of pills labeled as 5 (placebo) pills used in the trial had no desired biological activity and the V5 pills appeared to have clear benefit in terms of ameliorating baseline values. The trial is currently ongoing at the National Cancer Center, Ulaanbaatar, Mongolia and, provided additional funding is forthcoming, is expected to be completed by the end of 2021 .

\section{Effect of V5 on T cells from healthy donors}

This in vitro study was undertaken to elucidate the effect of short exposure ( $48 \mathrm{~h}$ ) of a physiologically relevant dose of V5 (diluted one million-fold, i.e., $0.1 \mu \mathrm{g} / \mathrm{mL}$ of cell culture medium) on effector $\mathrm{T}$ cells, either CD4- or CD8-positive T lymphocytes. The cells from the same donor were incubated in an identical culture medium without any V5 and they served as controls. This experiment was repeated three times on venous blood samples from unrelated healthy donors. The results from a representative flow cytometry analysis are shown in Figure 1. While limited, the data reveal an effect that has never been reported before. 


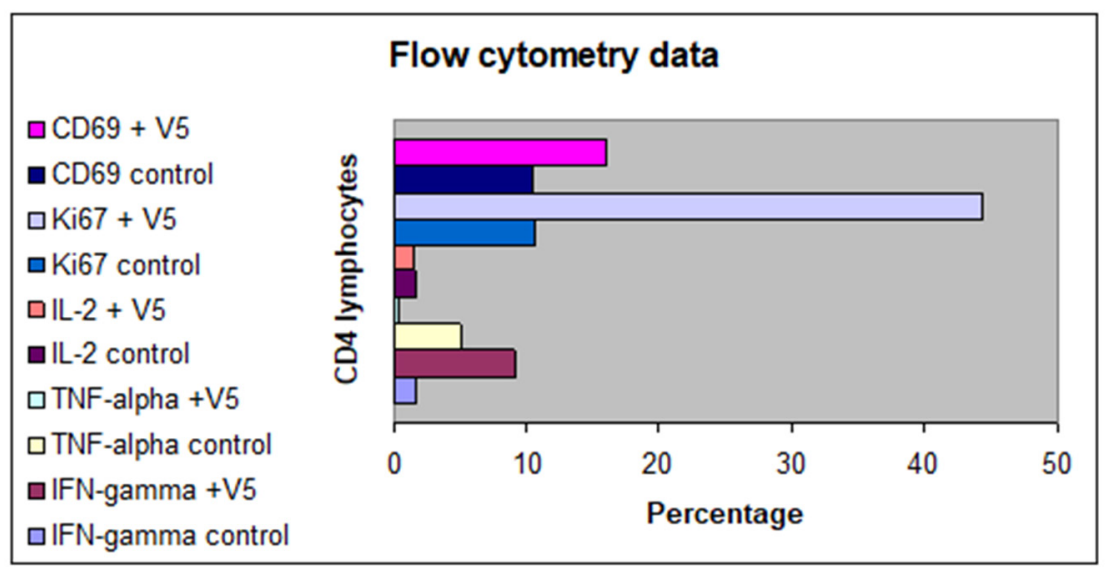

Figure 1. Effect of 48-h in vitro incubation of CD4 T lymphocytes from healthy volunteers with $\mathrm{V} 5\left(10^{-6}\right.$ dilution) on expression of cell activation/proliferation markers, i.e., CD69 and Ki-67, and IL-2, TNF- $\alpha$, and IFN- $\gamma$. Controls are unstimulated T cells as analyzed by flow cytometry at the same time. Except IL-2 expression, all other parameters were statistically distinct in V5-treated cells vs. controls

It appears that V5, which contains pooled antigens, including numerous tumor antigens and neoantigens, hepatitis virus fragments, and alloantigens, all circulating in the blood of patients with HCC, produces potent immune activation, while also inducing the anti-inflammatory effect. Markers of activation, i.e., CD69 and Ki67, rose on average 1.5- and 4-fold, respectively, compared to unstimulated CD4 and CD8 cells. The levels of IL-2 expression did not change, but IFN- $\gamma$ expressing cells increased six-fold. Remarkably, the inflammation marker TNF- $\alpha$ decreased almost 10-fold compared to control. The response of CD8 cells to V5 followed the same pattern as seen with CD4 cells, except expression of IFN- $\gamma$, which did not change compared to controls.

\section{DISCUSSION}

This paper provides interim, select data from an ongoing placebo-controlled, randomized Phase III trial of hepcortespenlisimut-L (Hepko-V5 or V5), which has successfully passed an open label, one-arm Phase II, 75-patient retrospective trial published in $2017^{[7]}$. Extensive preclinical studies started in 2002 have shown that V5 does not contain harmful chemical ingredients or live virus; does not cause cytotoxicity, mutagenicity, teratogenicity, or genotoxicity; and is not toxic to animals after acute or chronic exposure. V5 is very safe and remarkably well tolerated by healthy individuals as well as those with hepatitis or tuberculosis ${ }^{[8-10]}$. V5 intake increases body weight, improves the quality of life, and produces desired clinical benefit with improved liver function. The latest studies have shown that the beneficial effect is also observed in patients with HCC. As a rule, patients who received V5 experienced improvement in their baseline symptoms in as short as one week. Nevertheless, V5 has to be subjected to well-controlled randomized clinical study, such as outlined in this manuscript, to confirm that this intervention holds promise as a safe and effective immunotherapeutic modality for HCC indication.

Liver cancer is the fifth most commonly occurring cancer in men and the ninth most commonly occurring cancer in women. There were over 840,000 new cases in 2018 and prognosis is very poor. Mongolia firmly occupies the first place among all countries when it comes to incidence of HCC, while China has the largest number of patients with HCC, over $50 \%$ of all patients with $\mathrm{HCC}^{[11]}$. The incidence of HCC is expected to rise in the United States and across the world, given the increasing prevalence of hepatitis $\mathrm{C}$ and $\mathrm{B}$ infections, alcohol consumption, NASH, diabetes, toxin exposure, and obesity. By the time diagnosis of HCC is made, various surgical interventions or liver transplantation are often not feasible. While we have witnessed a surge of systemic therapies in recent years, the need for safer and more effective treatment remains. 
Hepcortespenlisimut-L represents an entirely novel class of immunotherapy that has no analogs among approaches being developed currently ${ }^{[12,13]}$. This approach is radically different from the checkpoint inhibitor class of immunotherapies, aimed to revive and keep the "weakened" immune system active. This approach is also different from classical injectable cancer vaccines that incorporate tumor antigens common in this type of cancer, such as $\mathrm{AFP}^{[14]}$, Glypican-3 expressed in $>80 \%$ of $\mathrm{HCC}^{[15]}$, or other multiepitope tumor peptides ${ }^{[16]}$. Considering that checkpoint inhibitors have low clinical benefit and singleor few-antigen vaccines have not delivered the expected success, it is clear that new approaches are still needed.

Scientists at Arizona State University recently identified more than 200,000 cancer neoantigens, which could lead to the development of broad-spectrum cancer vaccines ${ }^{[17]}$. V5 inherently incorporates all circulating antigens from pooled blood of HCC patients, including tumor unrelated immunogens such as viral hepatitis antigens and very large number of alloantigens, which are not necessarily pathogenic. It is clear that the identification of these antigens is a task posing enormous challenges. Taking aside the academic pursuit of finding the mechanism of action, this challenge is not very important to a patient who needs to be treated immediately, not after the putative antigen(s) are identified.

It is clear that oral delivery route is advantageous since this route eliminates the undesirable crossreactivity plaguing injectable vaccines. Another advantage associated with transmucosal passage of antigens is induction of the immune tolerance - a phenomenon we experience on a daily basis when we ingest, for example, food, which is composed of non-self, foreign antigens that would normally provoke violent immune reaction if delivered parenterally ${ }^{[18]}$. The gut immunity has evolved and differs from the systemic immunity by this critically distinct trait. We would not exist as a species if we did not develop the oral tolerance.

Our flow cytometry data, despite a limited number of studied parameters, suggest that the immune tolerance is not a passive state akin to immune anergy, but rather it is a very active process with the phenomenal activation of effector cells, as demonstrated by several-fold increase of immune activation markers such as Ki67 and CD69 in as little as $48 \mathrm{~h}$. At the same, time this process displays pronounced anti-inflammatory activity, as revealed by ten-fold decrease of TNF- $\alpha$, and no effect on IL-2 expression - the hallmark of T-cell activation. Increase in IFN- $\gamma$ output in CD4 but not in CD8 T cells was another unanticipated result. Thus, the exposure to a pool of antigens derived from a tiny amount of peripheral blood of patients with HCC produced results that have not been previously described in the literature.

The field of immunotherapy, particularly for oncology, is evolving rapidly, bringing new surprises daily. Recently, a study was published in Nature by Alspach et al ${ }^{[19]}$, in which they gave more weight to the role of helper CD4 lymphocytes in immuno-oncology over the usual culprit, i.e., CD8 killer cells. Whether V5 more affects CD4 rather than CD8 cells remains to be established; it is likely that both subpopulations are required, and that other subsets of immune cells are also critically involved ${ }^{[20,21]}$.

Our investigation of hepcortespenlisimut-L is in its early stage and we are sure we will make more surprising discoveries down the road. Besides the profound influence on the immune response, which is usually expected from cancer vaccines, we see very clear clinical benefits in terms of improved liver function and decrease in AFP levels, which we have shown to be correlated with tumor shrinkage. We have successfully used V5 since 2010 in hundreds of patients with HCC and we are confident that the outcome from this Phase III trial will be consistent with the results of the Phase II retrospective study published in $2017^{[7]}$. In conclusion, our experience in dealing with V5 and patients with HCC receiving this simple to use immunotherapy is supported by the present observation. Further studies will shed more light as to the mechanism of action and extent of the clinical benefit. 


\section{DECLARATIONS}

\section{Acknowledgments}

No professional writing services were used in writing up the manuscript. Authors acknowledge all patients who volunteered to participate in this study. Supporting medical staff and laboratory personnel are thanked for their help and support to make study happen.

\section{Authors' contributions}

Made substantial contributions to conception and design of the study and performed data analysis and interpretation: Bourinbaiar AS, Chinburen J, Batchuluun P, Munkhzaya C, Oyungerel D, Dandii D, Tsogkhuu H, Kutsyna GA, Tarakanovskaya MG, Bain AI, Jirathitikal V

Performed data acquisition, as well as provided administrative, technical, and material support: Reid AA, Borisova $\mathrm{V}$

\section{Availability of data and materials}

The data will be shared upon completion and publication of this study in full.

\section{Financial support and sponsorship}

This study would never have had a chance to succeed if we did not receive the financial contribution from our former patient with terminal HCC, Dr. Steve Kramer - now in complete remission since 2014 - and his spouse Jane Kramer, who as our genuine friends generously supported our effort over many years. Additional support was provided by several other patients who contributed extra in addition to regular payment for Hepko-V5. They were not involved in clinical study design, collection, analysis and interpretation of data.

\section{Conflicts of interest}

All authors, except officers of Immunitor company, i.e., Bourinbaiar AS, Bain AI, Jirathitikal V, Borisova V, Reid AA, declare that there are no conflicts of interest.

\section{Ethical approval and consent to participate}

The study was approved by the Institutional Review Board of Immunitor LLC (\#IRB00010671) and conducted in accordance with the declaration of Helsinki in conformity with good clinical practice as defined by the International Conference on Harmonization. Further information about this study is available at the ClinicalTrials.gov website under ID: NCT02232490.

\section{Consent for publication}

Not applicable.

\section{Copyright}

(c) The Author(s) 2020.

\section{REFERENCES}

1. Petrick JL, Florio AA, Znaor A, Ruggieri D, Laversanne M, et al. International trends in hepatocellular carcinoma incidence, 19782012. Int J Cancer 2019; Epub ahead of print doi: 10.1002/ijc.32723.

2. Rawla P, Sunkara T, Muralidharan P, Raj JP. Update in global trends and aetiology of hepatocellular carcinoma. Contemp Oncol (Pozn) 2018;22:141-50.

3. Ringelhan M, Pfister D, O’Connor T, Polansky E, Heikenwalder M. The immunology of hepatocellular carcinoma. Nat Immunol 2018;19:222-32.

4. Pinter M, Peck-Radosavljevic M. Review article: systemic treatment of hepatocellular carcinoma. Aliment Pharmacol Ther 2018;48:598-609.

5. Llovet JM, Montal R, Sia D, Finn RS. Molecular therapies and precision medicine for hepatocellular carcinoma. Nat Rev Clin Oncol 2018;15:599-616. 
6. Longo V, Brunetti O, Gnoni A, Licchetta A, Delcuratolo S, et al. Emerging role of immune checkpoint inhibitors in hepatocellular carcinoma. Medicina (Kaunas) 2019;55:E698.

7. Tarakanovskaya MG, Chinburen J, Batchuluun P, Munkhzaya C, Purevsuren G, et al. Open-label Phase II clinical trial in 75 patients with advanced hepatocellular carcinoma receiving daily dose of tableted liver cancer vaccine, hepcortespenlisimut-L. J Hepatocell Carcinoma 2017;4:59-69.

8. Batdelger D, Dandii D, Jirathitikal V, Bourinbaiar AS. Open-label trial of therapeutic immunization with oral V-5 Immunitor (V5) vaccine in patients with chronic hepatitis C. Vaccine 2008;26:2733-7.

9. Batdelger D, Dandii D, Dahgwahdorj Y, Erdenetsogt E, Oyunbileg J, et al. Clinical experience with therapeutic vaccines designed for patients with hepatitis. Curr Pharm Des 2009;15:1159-71.

10. Bourinbaiar AS, Mezentseva MV, Butov DA, Nyasulu PS, Efremenko YuV, et al. Immune approaches in tuberculosis therapy: a brief overview. Exp Rev Anti-infect Ther 2012;10:381-9.

11. Bray F, Ferlay J, Soerjomataram I, Siegel RL, Torre LA, et al. Global cancer statistics 2018: GLOBOCAN estimates of incidence and mortality worldwide for 36 cancers in 185 countries. CA Cancer J Clin 2018;68:394-424.

12. Zongyi Y, Xiaowu L. Immunotherapy for hepatocellular carcinoma. Cancer Lett 2019;470:8-17.

13. Baradaran Noveiry B, Hirbod-Mobarakeh A, Khalili N, Hourshad N, Greten TF, et al. Specific immunotherapy in hepatocellular cancer: a systematic review. J Gastroenterol Hepatol 2017;32:339-51.

14. Butterfield LH, Economou JS, Gamblin TC, Geller DA. Alpha fetoprotein DNA prime and adenovirus boost immunization of two hepatocellular cancer patients. J Transl Med 2014;12:86.

15. Nobuoka D, Yoshikawa T, Sawada Y, Fujiwara T, Nakatsura T. Peptide vaccines for hepatocellular carcinoma. Hum Vaccin Immunother 2013;9:210-2.

16. Buonaguro L; HEPAVAC Consortium. Developments in cancer vaccines for hepatocellular carcinoma. Cancer Immunol Immunother 2016;65:93-9.

17. Shen L, Zhang J, Lee H, Batista MT, Johnston SA. RNA transcription and splicing errors as a source of cancer frameshift neoantigens for vaccines. Sci Rep 2019;9:1-3.

18. Silin DS, Lyubomska OV, Jirathitikal V, Bourinbaiar AS. Oral vaccination: where we are? Expert Opin Drug Deliv 2007;4:323-40.

19. Alspach E, Lussier DM, Miceli AP, Kizhvatov I, DuPage M, et al. MHC-II neoantigens shape tumour immunity and response to immunotherapy. Nature 2019:574:696-701.

20. Hilmi M, Neuzillet C, Calderaro J, Lafdil F, Pawlotsky JM, et al. Angiogenesis and immune checkpoint inhibitors as therapies for hepatocellular carcinoma: current knowledge and future research directions. J Immunother Cancer 2019;7:333.

21. Rafei H, Mehta RS, Rezvani K. Editorial: cellular therapies in cancer. Front Immunol 2019;10:2788. 